\title{
Disparidades salariales y la tasa interna de retorno a la educación privada en los docentes de la Universidad del Cauca ${ }^{1}$
}

\author{
Wage disparities and internal rate of return to private \\ education teachers at the University of Cauca
}

Recibido: 29 de septiembre de 2014 - Revisado: 26 de febrero de 2015 - Aceptado: 08 de abril de 2015

\author{
Andrés Mauricio Gómez Sánchez ${ }^{2}$ \\ Zoraida Ramírez Gutiérrez ${ }^{3}$
}

\section{Resumen}

Este documento tiene por objetivo encontrar la tasa interna de retorno a la educación privada de los docentes de la Universidad del Cauca (Popayán, Colombia) controlada por un conjunto de variables sociales y económicas. Para lograrlo, se construyen modelos econométricos exentos de problemas de endogeneidad y sesgos de selección muestral, ya que incluye la productividad académica del docente como variable instrumental y adicionalmente se cuenta con información poblacional. Los resultados evidencian, entre muchos otros, que en general la tasa de retorno es baja para los docentes de la universidad en comparación con las halladas para toda Colombia, y como ya es un patrón nacional y mundial, dicha tasa es inferior para las mujeres que para los hombres.

\section{Palabras clave}

Tasa interna de retorno, educación, capital humano, Universidad del Cauca, ecuaciones de Mincer, econometría.

\footnotetext{
Abstract

This document aims to find the internal rate of return to private education from teachers of Universidad del Cauca (Popayan, Colombia) controlled by a set of social and economic variables. To achieve this, econometric models, exempt of endogeneity problems and sample selection biases, are built, as it includes academic productivity of teachers as an instrumental variable and additionally has population data. The results show, among many others, that the overall rate of return is lower for university professors compared with those found for all Colombia, and as it is a national and global pattern, this rate is lower for women than for men.

\section{Keywords}

Internal rate of return, education, human capital, University of Cauca, Mincer equations, econometrics.
}

${ }^{1}$ El artículo resultado del proyecto de investigación "Disparidades salariales de los docentes en la Universidad del Cauca, 2014". Se agradece a la Vicerrectoría Administrativa de la Universidad del Cauca, Popayán, Colombia; por facilitar la información para este estudio.

2 Magíster en Economía Aplicada, Universidad del Valle, Cali, Colombia. Especialista en Gerencia de Proyectos, Universidad del Cauca, Popayán, Colombia. Economista, Universidad del Valle. Profesor titular, Departamento de Ciencias Económicas, Universidad del Cauca. Miembro del Grupo de Investigación Entropía, Universidad del Cauca, Popayán, Colombia. Correo electrónico: amgomez@unicauca.edu.co

${ }^{3}$ Magíster en Administración Econó mica y Financiera, Universidad Tecnológica de Pereira. Especialista en Administración Financiera, Universidad La Gran Colombia. Contadora pública, Universidad del Quindío. Profesora asociada, Departamento de Contaduría, Universidad del Cauca, Popayán, Colombia. Coordinadora del Grupo de Estudio e Investigación en Finanzas y Gestión (GREIFG), Universidad del Cauca, Popayán, Colombia. Correo electrónico: zramirez@unicauca.edu.co

Para citar este artículo: Gómez, A., \& Rámirez, Z. (2015). Disparidades salariales y la tasa interna de retorno a la educación privada en los docentes de la Universidad del Cauca. Revista Civilizar Ciencias Sociales y Humanas, 15(28), 165-180. 


\section{Introducción}

La disposición de las diferencias salariales entre individuos es uno de los temas centrales de estudio de la economía neoclásica en las últimas décadas, donde la aplicación del modelo de capital humano es bien respaldado por los resultados empíricos.

El concepto de capital humano en su definición genérica hace referencia a todo el cúmulo no solo de conocimientos o formación, sino también de habilidades, destrezas, experiencias y demás atributos que se encuentran presentes en los individuos (Salas, 2008). Dicho cúmulo determina la productividad y por tanto los niveles salariales de los sujetos, encontrándose que a mayor inversión en capital humano mayores salarios y viceversa. En el campo institucional es importante la presencia de altos niveles de capital humano porque se eleva la competitividad de las empresas o instituciones, lo que se constituye en una herramienta diferenciadora de competencia frente a sus rivales en el mercado.

Las universidades como cualquier otra entidad o empresa también están inmersas en esta dinámica, ya que la consolidación en la formación académica e investigativa, y la adquisición de habilidades y/o destrezas educativas por parte de sus profesores, es un factor clave a la hora de participar en el mercado de la educación; porque esto significa en general un incremento en la calidad de los programas que se ofrecen a la comunidad. De manera individual para los docentes, mayores cualificaciones y experiencia académica, les permite mejorar la calidad de la docencia directa, ingresar, impulsar y fortalecer grupos de investigación, acrecentar las publicaciones (libros, artículos y ponencias), crear y coordinar nuevos programas de posgrado, afianzar su reconocimiento público, etc., lo que redunda en niveles salariales superiores.

En este orden de consideraciones, el presente documento intenta hallar la tasa interna de retorno a la educación privada de los profesores de la Universidad del Cauca (Colombia), a la luz de un conjunto de características sociales y económicas de los mismos, como un aporte más a la gran cantidad de estudios aplicados que en materia de capital humano se han realizado en el ámbito nacional e internacional. Los elementos diferenciadores de este esfuerzo radican en la inexistencia de un análisis de este tipo para Popayán y el Cauca, y en que tal análisis no se ha llevado a cabo por lo menos para nuestro país en la esfera de las universidades públicas o privadas.

\section{Visiones disímiles del capital humano}

El concepto de capital humano se debe a Schultz (1959), quien lo entiende como una inversión que efectúan los individuos pero no en documentos de deuda pública o privada, sino en ellos mismos y, por ende, tiene costos a corto plazo pero beneficios o ganancias en el largo. Para Becker (1993) es una inversión en educación $\mathrm{y}$ en formación en el sitio de trabajo, sinónimo de niveles superiores de productividad.

En la actualidad la definición cambia dependiendo de la perspectiva o escuela de pensamiento, pero la más generalizada dice que debe interpretarse como el conjunto de conocimientos, habilidades, experiencias y demás atributos que están presentes en los individuos (Salas, 2008) y que marcan los diferenciales salariales entre ellos. Es importante resaltar que las inversiones hechas por los padres en la crianza y educación de sus hijos también se consideran capital humano, por lo que no es un concepto únicamente ligado a la educación como tal, pues puede provenir del hogar y antes del inicio o finalización de la etapa escolar.

De acuerdo con la Organización para la Cooperación y el Desarrollo Económicos (OCDE) (1998), existen tres enfoques para medir el capital humano. Por los niveles de estudios de los adultos, por la evaluación de sus 
habilidades y por sus salarios. Según la primera visión, la medida viene dada por el grado de estudio más alto alcanzado por el individuo, dejando de lado otras formas de capital como la experiencia, por ejemplo y adicionalmente solo miden la cantidad pero no la calidad de la educación.

Para el segundo enfoque, se valoran las habilidades y destrezas de los sujetos a través de una encuesta aplicada por la International Adult Literacy Survey (IALS) a personas entre los 16 años y los 65 años de edad evaluando la suficiencia en capacidad lectora y matemáticas, pero también en procesar información, comunicación con fluidez, etc. El tercer enfoque estima que la educación, experiencia, habilidades y destrezas -es decir, el stock del capital humano-, determina los salarios y sus diferenciales entre individuos.

Esta última visión es la que tiene en cuenta un gran conjunto de escuelas de pensamiento económico, debido a que se ha comprobado de manera empírica la relación directa entre capital humano y salarios. En algunos casos las formas teóricas como se consolida este vínculo, tienen sutiles diferencias entre una y otra escuela, pero en otros casos existen grandes distancias.

En la teoría pura del capital humano, Becker (1964) afirma que los ingresos van de la mano con la edad del individuo y más exactamente con la etapa de su ciclo vital. Por tanto, una persona joven con poca experiencia tiene salarios bajos, pero aquella que posee un grado de madurez más alto y mayor experiencia, logra el máximo de ingresos en la etapa cumbre de su ciclo de vida, aunque una vez comienza a envejecer, los ingresos declinan porque su productividad laboral e intelectual decrece.

Valelapenaaclararque el comportamiento cíclico es el mismo en el interior de un grupo, pero difiere para los individuos que tienen distintos niveles de estudio, esto es, entre grupos. Los estudios aplicados por lo común fallan en la implementación de esta teoría porque con frecuencia se basan en datos de corte transversal, lo que impide mostrar la evolución de los salarios en el mediano y largo plazo, pues solo dan una idea de lo que sucede con los sujetos en un momento en el tiempo. Por ende, se requieren datos panel para poder ser fieles a la teoría, pero la insuficiencia en los países de información de esa clase para estos casos, dificulta que los estudios sean completos.

En el campo de los modelos econométricos debe resaltarse el modelo propuesto por Mincer (1974). Básicamente allí se estima la relación existente entre los ingresos laborales (salarios) y los niveles de educación y experiencia laboral de los individuos, estas últimas medidas en años. Para capturar la concavidad de la función, se agrega el cuadrado de los años de experiencia; es decir, que se captura si el aporte de un año adicional aumenta más que proporcionalmente el ingreso laboral (Cárdenas, 2007). El coeficiente que acompaña a la variable educación representa en cuanto se eleva porcentualmente, los salarios si se incurre en un año más de escolaridad, por esta razón se denomina tasa interna de retorno de la educación. Este modelo se conoce en la literatura del capital humano como la "ecuación minceriana" -en honor a su proponente, Jacob Mincer-, la cual se estima por mínimos cuadrados ordinarios $(\mathrm{MCO})^{1}$.

Se aclara que de acuerdo con Heckman, Lochner y Todd (2005) para que dicho parámetro se interprete en estricto de esta manera, se debe cumplir una serie de supuestos difíciles de mantener empíricamente. En primera instancia, el logaritmo de los salarios debe ser lineal frente a la educación, todos los individuos deben enfrentar una vida laboral con la misma duración, no deben existir costos económicos o psicológicos de la educación, ni tampoco impuestos al ingreso. Por la imposibilidad de 
su cumplimiento, tales autores recomiendan interpretar el parámetro no como la tasa interna de retorno privada a la educación, sino más bien como la tasa de crecimiento del salario frente a un año adicional de educación.

Otra teoría asociada al concepto de capital humano es la del credencialismo. Autores como Arrow (1973), Spence (1973) y Stiglitz (1975) sostienen que debido a la información imperfecta y asimétrica, las empresas deben acudir a un conjunto de señales que sirve de medida de su capacidad y productividad. Por tal razón, los trabajadores más hábiles y mejor preparados (quienes tienen un mayor número de posgrados por ejemplo) emiten señales más fuertes y claras que otros, por lo que son contratados con unos salarios superiores respecto a los demás.

La escuela institucionalista, por otro lado, plantea que la productividad de los trabajadores se logra al interior de las empresas y no por fuera de ellas. Doeringer y Piore $(1971,1983)$ y Thurow $(1975,1983)$ hacen una digresión entre los niveles de educación, la experiencia y la productividad argumentando que los trabajadores obtienen salarios más altos, porque dado sus niveles de educación son asignados a cargos laborales donde estos explotan todo su conocimiento, alcanzando una mayor experticia y por tanto mejores ingresos.

La corriente de la dualización del mercado (Reich, Gordon, \& Edwards, 1973) considera que el mercado laboral se segmenta en dos: el primario y el secundario. En el primario se encuentran los mejores empleos, los mejor remunerados y los de mayor responsabilidad, por lo que se requiere personal más capacitado. En el segmento secundario, se hallan los empleos que requieren personal poco capacitado, con responsabilidades simples y, por ende, con bajas remuneraciones.

Una visión diferente a la precedente, sostiene que los trabajadores hacen fila o "cola" para llegar a acceder a los cargos más altos en las organizaciones, lo cual se consigue en el mediano y largo plazo. Por tanto, al inicio los cargos no son los más altos y las remuneraciones tampoco, pero en la medida que pasa el tiempo, se acumula experiencia y en algunos casos años de estudio que permiten ir escalando hasta lograr los puestos más apetecidos.

Por último, la escuela marxista plantea de manera distinta la relación entre ingresos y educación. Los ingresos básicamente dependen de la inteligencia y del origen social y económico del trabajador. En efecto, Bowles y Gintis (1976, 2006) afirman que la dualidad entre educación y productividad no proviene de la adquisición de cualificaciones, sino de la reproducción de la estructura de clases de la sociedad, por lo que el efecto de la educación sobre los ingresos no es relevante, pues lo que interesa es el coeficiente intelectual y la procedencia socioeconómica del individuo.

\section{Aplicaciones al caso colombiano}

Los estudios empíricos de la teoría del capital humano tienen amplias aplicaciones internacionales y nacionales. En general, los estudios capturan la tasa interna de retorno de la educación en Colombia y las variantes obedecen a las bases de datos utilizadas, las variables que se emplean para tener en cuenta los posibles sesgos y los métodos econométricos.

Un primer estudio destacado es el de Maldonado (2000), que implementa el modelo de Roy para medir la magnitud de los diferenciales salariales entre los trabajadores del sector público y privado para Colombia en 1997. La utilización del modelo de Roy permite eliminar el sesgo de selección para sectores separados y a su vez ayuda a realizar predicciones sobre lo que un trabajador ganaría en otro sector. El autor usa la información suministrada por la Encuesta Nacional de Hogares (ENH) del año 1996 para estimar modelos probit que capturan 
la probabilidad de trabajar en el sector público o privado, considerando como variables explicativas al género, el estado civil y la experiencia (entre otras) y luego pasar a estimar la lambda de Heckman e incluirla en la ecuación de ingresos (para hombres y mujeres por separado) y corregir así el sesgo de selección. Los resultados muestran que los salarios de los trabajadores del sector privado son inferiores respecto a sus similares del sector público, aspecto que no se cumple con los trabajadores que tienen posgrado.

La investigación de Casas, Gallego y Sepúlveda (2003) explica los retornos a la educación en Colombia basándose en la teoría del capital humano, pretendiendo resaltar la existencia y magnitud del sesgo por habilidad en la ecuación de Mincer. Para tal efecto se utiliza la Encuesta de Calidad de Vida 1997 (ECV97) y la encuesta del Instituto Ser de Investigación del año 1997.

Los resultados arrojan que desde la encuesta Ser el sesgo es solo del 1,2 \% en los retornos a la educación, mientras que con la ECV97, los retornos están en el rango entre el 8 $\%$ y el $10 \%$, resultado similar a los hallados por los estudios pioneros de Psacharopoulos y Vélez (1992) y Tenjo (1993). Los autores concluyen que a pesar de que hay un sesgo positivo, este no altera ampliamente los resultados de la tasa de retorno de la educación en Colombia, ya que la diferencia es muy pequeña cuando no se tiene en cuenta el sesgo.

En otra variante de los análisis adelantados al interior del país, se tiene el de Prada (2006), el cual indaga, a diferencia de los anteriores, por la dinámica de los retornos de la educación en Colombia entre 1985 y 2000 . La metodología econométrica incluye regresiones por percentiles y una ecuación de salarios con splines lineales, debido a que los retornos de un año adicional de educación no son idénticos a través de todos los niveles de escolaridad. Los resultados manifiestan que los retornos varían a través de distintos niveles de escolaridad; así los retornos son altos para la educación secundaria; y con la educación universitaria son aún mayores.

Además se comprueba que los empleados con altos niveles educativos tienen un incremento mayor en la retribución de la educación, y que, como consecuencia de esto, el país conservó un aumento del sesgo de la distribución condicional del salario. El resultado más importante es la similitud de la evolución de los retornos de la educación universitaria de las mujeres frente a los hombres, a lo largo de la década de los noventa. Este hecho significa que las conexiones y posibilidades laborales reportan salarios semejantes para hombres y mujeres.

La investigación de García, Guataqui, Guerra y Maldonado (2009), intenta superar las críticas que rodean al coeficiente que representa la tasa de retorno a la inversión en educación de la ecuación de Mincer, apoyándose en el enfoque metodológico proporcionado por Heckman et al. (2005). El documento desarrolla un enfoque más estructurado que estudios previos nacionales de la estimación de dicha tasa controlando el sesgo de selección de una mejor forma, pues incluye medidas más precisas de los ingresos laborales e introduce el papel de los costos de la educación y los impuestos sobre la renta.

En términos puntuales, los autores encuentran una menor tasa de retorno $(7,4 \%)$ que la que se halla en la literatura colombiana $(12,8 \%)$, aunque los resultados varían según el año en análisis y el sexo del individuo; hecho que refuerza las consideraciones respecto a la discriminación de género en el mercado laboral colombiano. En general, los resultados muestran que la educación superior genera rendimientos positivos, pero existe una brecha de género y un efecto negativo de los impuestos sobre los rendimientos de la inversión en educación. 
En un trabajo más reciente, Vargas (2013) calcula los retornos a la educación de los trabajadores en Colombia, pero al contrario de las investigaciones precedentes se centra en las zonas donde se educaron los individuos. Observa entonces las diferencias en los retornos de las personas que estudiaron y trabajan en las ciudades frente a aquellas que hicieron lo mismo pero en el campo, al igual que para otro conjunto denominado migrantes.

A la ecuación de Mincer tradicional se le agregan variables como el género, la pertenencia a redes sociales, los años de educación de los padres para controlar el sesgo de habilidad y otras de tipo dicotómico que descomponen los años de educación en niveles. Se evidencia que los retornos son más altos para las personas que tienen cada vez mayores años de estudio, y por regiones se muestra que para los individuos que se formaron y residen en las ciudades la tasa supera al $1 \%$, para los migrantes tasas inferiores al $1 \%$, y para los que se educaron y residen en el campo las tasas son muy cercanas al $0 \%$.

\section{Hechos estilizados del capital humano en la Universidad del Cauca ${ }^{2}$}

La Universidad del Cauca cuenta hasta abril 30 de 2014 con un total de 570 profesores y profesoras, distribuidos en 53 programas de pregrado, incluyendo el programa de formación en inglés (PFI), los cuales conforman un total de nueve facultades. Los docentes que ocupan cargos administrativos como rectoría, vicerrectorías, decanaturas, dirección de centros, entre otros, son incluidos en sus respectivos departamentos.

La edad media de los docentes es de 50,5 años y su experiencia laboral promedio al interior de la universidad es de 17 años, aunque los profesores que tienen una experiencia inferior a dicho promedio alcanzan el 61,2\%, cifra superior a aquellos que están por encima $(38,8 \%)$. Esto indica que posiblemente los maestros ingresan alrededor de los treinta años a la planta docente y gran parte de ellos están debajo de la edad promedio.

Los niveles salariales más altos encuentran una relación directa con la edad del docente, de tal forma que los programas que presentan profesores más longevos son aquellos cuyos ingresos salariales son los más elevados. Quizá esto obedezca a que una mayor trayectoria permite tener mayores niveles de formación, experiencia y productividad académica. Dicha tendencia no aplica para muy pocos departamentos cuyos niveles salariales son altos pero sus edades están muy por debajo del promedio. Esto indica que la formación docente y la productividad académica ha sido más rápida y agresiva aquí que en los demás departamentos de la universidad.

Respecto a los grados de formación obtenidos, se encuentra que los niveles más frecuentemente alcanzados en los docentes son las especializaciones y los que menos son los doctorados y posdoctorados. En efecto, como lo muestra la gráfica 1 , el 65,6\% de los docentes tiene posgrado en especializaciones, y casi la mitad de los maestros tiene estudios de maestría $(51,8 \%$ ), pero ya de manera distante se encuentra la formación doctoral, la cual solo consigue el $17 \%$ aproximadamente en los profesores y casi nula es la formación posdoctoral $(0,2 \%)$.

Cuando estas cifras se cruzan con la edad, se muestra que son aquellos educadores más longevos quienes tienen los niveles posgraduales más bajos, por lo que posiblemente la tendencia cambie no solo cuando se realice un relevo generacional, sino también cuando a los nuevos docentes se les exija un nivel de formación más alto. 
Gráfica 1.

Niveles de formación de los docentes de planta. Universidad del Cauca, abril de 2014

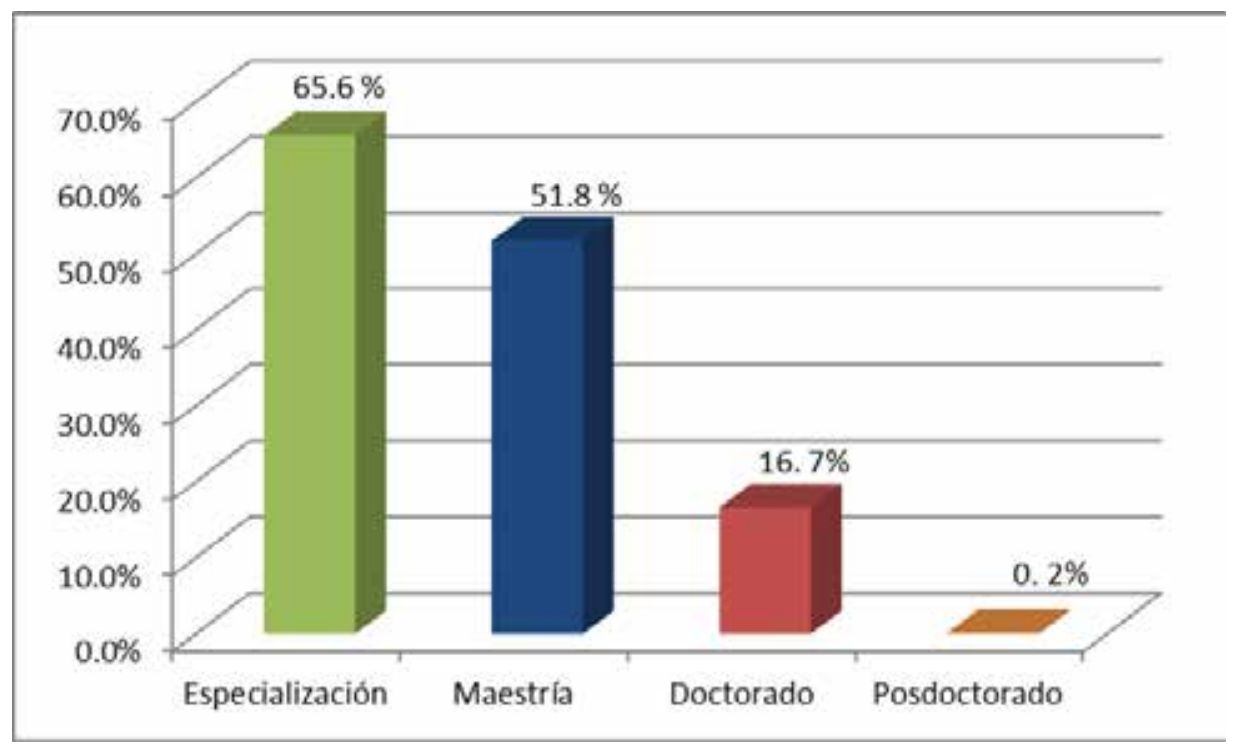

Fuente: elaboración propia.

Del total de docentes se advierte una mayor proporción de profesores que de profesoras, toda vez que los hombres doblan a las mujeres en número, alcanzando una participación del $68,4 \%$, mientras que el restante $31,6 \%$ son mujeres. Las profesoras tienen mayor participación en departamentos ligados a la medicina (fisioterapia, enfermería, pediatría) y otras como educación, comunicación o estudios interculturales, pero los profesores tienen más alta presencia en carreras relacionadas con la ingeniería, la matemática, la economía, el derecho y la ciencia política.

Los niveles educativos controlados por género, evidencian que independientemente del grado de formación al que se aluda, las profesoras tienen menos formación que los profesores. En este orden de ideas, por cada especialista mujer en la universidad hay dos especialistas hombres, hecho que se repite en las maestrías. La situación más notable se observa en los doctorados, ya que por cada profesora con este título, existen tres profesores con la misma educación.
Lo anterior a manera de hipótesis posiblemente sea a causa del rol que asumen las mujeres en general en los hogares. Si bien es cierto que las mujeres más preparadas académicamente generan mayores niveles de independencia personal, social y económica, su papel de madres, esposas y las labores hogareñas que endilga una sociedad patriarcal como la de nuestro país, les podría estar impidiendo consolidar su formación académica. Por vía contraria los profesores pueden llegar a tener más oportunidades para conseguir más y mejores niveles educativos.

El escenario de la discriminación no se considera en este punto, toda vez que las oportunidades que brinda la universidad para adquirir niveles superiores de estudio están abiertas para ambos sexos. Adicionalmente, con la información suministrada no se puede hablar con certeza de que exista el fenómeno. Por el lado de la experiencia docente, se encuentra que las mujeres tienen menos que los hombres, siendo mayor la de estos últimos en alrededor de dos años. A nivel agregado, la experiencia alcanza los 17 años. 


\section{Modelación econométrica ${ }^{3}$}

Teniendo en cuenta la recomendación de Heckman et al. (2005) para capturar ya no los retornos a la educación, sino más bien la tasa de crecimiento de los salarios por años adicionales de educación en la población en análisis, se utilizará de acuerdo con Johansen (1992) el criterio de Pantula, es decir, se partirá del modelo más simple o parsimonioso al más complejo o menos parsimonioso, con el fin de seguir la evolución del sesgo en el retorno.

Todos los estudios aplicados parten de la ecuación clásica de Mincer (1974), la cual plantea que los niveles salariales de un individuo expresados en logaritmo ( $L n w)$, se explican por los niveles educativos medidos en años $(E d u)$ y por la experiencia laboral $(\operatorname{Exp})$ también medida en años. Para determinar los rendimientos decrecientes de los ingresos frente a un año adicional de experiencia (función cóncava), se incluye dicha variable de forma cuadrada $\operatorname{Exp}^{2}$. Así, la ecuación es la que muestra la fórmula (1).

$\operatorname{Ln}\left(w_{i}\right)=\beta_{1}+\beta_{2} E d u_{i}+\beta_{3} \operatorname{Exp}_{i}+\beta_{4} \operatorname{Exp}_{i}^{2}+U_{i}$

El parámetro que acompaña a la variable de educación muestra en qué proporción se elevan los salarios por cada año de formación de la persona. Se espera que el parámetro sea positivo toda vez que a mayor nivel de estudio, mayor productividad y por tanto, superiores niveles de ingreso. Para la experiencia se espera el mismo signo por iguales razones. Para el último parámetro, su valor debe ser negativo si la función es cóncava, esto es, un año más de experiencia incrementa el ingreso cada vez en una proporción menor frente al año anterior. Finalmente, $U i$ denota los términos aleatorios de error: aquellas variables que influyen en los niveles salariales del individuo pero que no se consideran de forma explícita en el modelo. Se asume que dicha variable cumple con los supuestos clásicos de media cero, homocedasticidad y no autocorrelación. Los resultados del modelo aplicado a la Universidad del Cauca se presentan en la tabla 1 .

Tabla 1.

Estimación modelo 1 con sesgo de variable omitida

\begin{tabular}{|l|c|c|}
\hline \multicolumn{1}{|c|}{ Variable } & Coeficiente & P-valor \\
\hline C & 14,079 & 0,000 \\
EDU & $4,17 \%$ & 0,000 \\
EXPE & $2,68 \%$ & 0,000 \\
EXPE^2 & $-0,02 \%$ & 0,118 \\
\hline Observaciones & \multicolumn{2}{|c|}{570} \\
\hline R2 & \multicolumn{2}{|c|}{0,2806} \\
\hline P-valor F & \multicolumn{2}{|c|}{0,0000} \\
\hline
\end{tabular}

Nota: *Estimaciones realizadas bajo consistencia White.

Fuente: elaboración propia.

Los resultados preliminares encuentran en términos estadísticos significancias individuales muy altas, sobre todo con la variable educación $\mathrm{y}$ experiencia, y todos los signos esperados son los correctos. La experiencia al cuadrado no es significativa por muy poco, y el valor del $R^{2}$ es del $28 \%$, que a pesar de ser bajo, es característico en estos modelos por la alta variabilidad en la información de corte transversal.

No obstante, el $P$-valor $F$ es igual a cero, mostrando que desde una perspectiva holística el modelo es confiable. En este orden de ideas, se puede decir que sin considerar el sesgo por variable omitida, el crecimiento de los salarios frente a la educación de los docentes es igual a 4,2 \%; por cada año de educación en el que incurran, ceteris paribus. Así mismo, en el caso de la experiencia alcanza $2,7 \%$ por año, y el incremento marginal es decreciente e igual al $0,2 \%$, dejando todo lo demás constante.

Como se sabe, estos resultados son sesgados e inconsistentes y de acuerdo con los autores anteriores, deben estar sobrestimando la verdadera tasa de crecimiento de los salarios. Para iniciar con la consideración de los sesgos hallados por Griliches (1977), se estima que los 
salarios expresados en logaritmos $-\ln (w)-$ se explican por los años de educación $(E d u)$, la experiencia $(\operatorname{Exp})$ y la experiencia al cuadrado $\left(\operatorname{Exp}^{2}\right)$ como en la ecuación minceriana ${ }^{4}$.

También se construye una variable de habilidad $(H a b)$ del profesor para eliminar el sesgo por variable omitida, la cual recoge la productividaden investigaciones, publicaciones, patentes y demás que han sido reconocidas como factor salarial mediante puntos ${ }^{5}$. Esto en el entendido que los docentes con mayores credenciales y experiencia académica son aquellos que desean y efectivamente pueden ingresar a estudios cada vez más altos ${ }^{6}$.

Ahora, para capturar mejor la varianza de los ingresos se agrega una variable dicotómica que recoge el género del profesor (Gen), la cual asume el valor de uno si es hombre y de cero si es mujer; ya que los y las docentes por sus diferentes roles en el hogar pueden tener más o menos posibilidades de incrementar su cualificación y productividad académica. En este orden, el modelo 2 es el que se observa en la ecuación (2).

$$
\begin{aligned}
\operatorname{Ln}\left(w_{i}\right)= & \alpha_{1}+\alpha_{2} E d u_{i}+\alpha_{3} \operatorname{Exp}_{i}+\alpha_{4} \operatorname{Exp}_{i}^{2}+\alpha_{5} \operatorname{Gen}_{i} \\
& +\alpha_{6} \operatorname{Hab}_{i}+\varepsilon_{i}(2)
\end{aligned}
$$

La variable $\varepsilon_{i}$ muestra los términos aleatorios de error: variables que influyen en los niveles salariales del docente pero que se consideran explícitamente en el modelo. Se asume que es bien comportada, es decir, tiene media cero, varianza constante y covarianza nula. Los resultados revelan que los signos esperados son los correctos y las significancias individuales son altas para todas las variables estimadas. El $R^{2}$ ahora es del $92 \%$ y el $P$-valor $F$ es cero, lo que prueba un modelo estadísticamente consistente. Por tanto, ahora incluyendo la variable que recoge la habilidad, se encuentra que por cada año de estudio, los docentes acrecientan sus salarios en 2,6\%, ceteris paribus. Este resultado es acorde con lo que señala Griliches (1977), ya que estima que si no se incluye dicha variable, se sobrestimaría la tasa.
Tabla 2.

Estimación modelo 2 sin sesgo de variable omitida

\begin{tabular}{|l|c|c|}
\hline \multicolumn{1}{|c|}{ Variable } & Coeficiente & P-valor \\
\hline C & 14,246 & 0,000 \\
EDU & $2,61 \%$ & 0,000 \\
EXPE & $0,74 \%$ & 0,003 \\
EXPE^2 & $-0.02 \%$ & 0,002 \\
GEN & $-1,69 \%$ & 0,022 \\
\hline HAB & $0,25 \%$ & 0,000 \\
\hline Observaciones & \multicolumn{2}{|c|}{570} \\
\hline R2 & \multicolumn{2}{|c|}{0,923} \\
\hline P-valor F & \multicolumn{2}{|c|}{0,000} \\
\hline
\end{tabular}

Nota: * Estimaciones realizadas bajo consistencia White.

Fuente: elaboración propia.

Por el lado de la experiencia, se tiene que la tasa de crecimiento salarios-educación es muy baja, ya que no alcanza el $1 \%$. La experiencia al cuadrado muestra rendimientos marginales decrecientes frente a esta variable $\mathrm{y}$ el género evidencia de manera sorpresiva, que los hombres por su condición disminuyen el salario en $1,69 \%$ al año, lo que desvirtúa de alguna forma la idea de discriminación por género en cuanto a salarios en la Universidad del Cauca. Finalmente la habilidad muestra que por cada punto reconocido por productividad, el ingreso salarial se amplía en 0,25\%.

Para dar cuenta de los otros sesgos que halló Griliches (1977), se estima la ecuación reducida a través del método de MCO. Siguiendo a Wooldridge (2001) las variables instrumentales que se emplearon para la ecuación reducida de la educación son las explicativas de la ecuación (2), más tres dicotómicas que recogen los niveles educativos del docente (especialización, maestría y doctorado), las cuales permiten menguar el sesgo de acuerdo con Sapelli (2009). La ecuación reducida es la que expone la fórmula (3).

$$
\begin{aligned}
E d u_{i}= & \delta_{1}+\delta_{2} \operatorname{Exp}_{i}+\delta_{3} \operatorname{Exp}_{i}^{2}+\delta_{4} \text { Gen }_{i}+\delta_{5} \mathrm{Hab}_{i} \\
& +\delta_{6} \mathrm{DEsp}_{i}+\delta_{7} \text { DMae }_{i}+\delta_{8} \text { Doc }_{i}+\mu_{i}
\end{aligned}
$$


Donde DEsp, DMae y DDoc son variables dicotómicas que asumen el valor de uno si el docente tiene estudios de especialización, maestría y doctorado; y de cero si no presenta dichos estudios, respectivamente. Con todo lo anterior, se obtiene el pronóstico de la variable educación $(E d u f)$, que es ya una variable depurada de cualquier otro tipo de influencia, esto es, totalmente exógena y se introduce en el modelo como variable explicativa. Por último y a la luz de Hausman (1978) se incluyen los términos de error estimados de la ecuación (3) para determinar si se presenta endogeneidad o no. El modelo es el de la ecuación (4).

$$
L n\left(w_{i}\right)=\gamma_{1}+\gamma_{2} E d u f_{i}+\gamma_{3} \operatorname{Exp}_{i}+\gamma_{4} \operatorname{Exp}_{i}^{2}+\gamma_{5} G_{e n}+\gamma_{6} H a b_{i}+\gamma_{7} \hat{\mu}_{i}+\pi_{i}
$$

Donde $\pi_{i}$ expresa los errores aleatorios, que se suponen bien comportados. Los resultados estimados por Mínimos Cuadrados en 2 Etapas -MC2E arrojan que los parámetros son estadísticamente significativos tanto para individuos como para conjuntos y sus signos son los esperados. La incorporación de la habilidad y la estimación por $M C 2 E$ elevan la expansión de los salarios frente a la educación si se compara con el caso precedente. Así, se puede advertir que por cada año de estudio, los profesores y profesoras de la Universidad del Cauca incrementan sus ingresos salariales en $3,5 \%$. Por experiencia lo hacen en $0,75 \%$; por habilidad en $0,25 \%$ y por ser hombres en 1,8 $\%$, todo en condiciones ceteris paribus.

Tabla 3.

Estimación modelo 2 sin sesgo

de variable omitida. $M C 2 E$

\begin{tabular}{|l|c|c|}
\hline \multicolumn{1}{|c|}{ Variable } & Coeficiente & P-valor \\
\hline C & 14,06 & 0,0000 \\
EDUF & $3,53 \%$ & 0,0000 \\
EXPE & $0,75 \%$ & 0,0024 \\
EXPE^2 & $-0,02 \%$ & 0,0027 \\
GEN & $-1,84 \%$ & 0,0173 \\
HAB & $0,25 \%$ & 0,0000 \\
RESID & $-0,53 \%$ & 0,3148 \\
\hline Observaciones & \multicolumn{2}{|c|}{570} \\
\hline R2 & \multicolumn{2}{|c|}{0,92} \\
\hline P-valor F & \multicolumn{2}{|c|}{0,0000} \\
\hline
\end{tabular}

Nota: $*$ Donde RESID $=$

** Estimaciones realizadas bajo consistencia White.

Fuente: elaboración propia.

Vale la pena mencionar que la inclusión de los residuales muestra que el problema de endogeneidad se ha solucionado, ya que resultan ser no significativos estadísticamente, lo cual va en concordancia con lo que establece la prueba de Hausman (1978). Por último, la estimación del crecimiento salarial respecto a la educación discriminada por género indica que este es inferior para las mujeres. En efecto, para las profesoras, cada año de educación genera $3,3 \%$ de aumento de salario, mientras que para los profesores es de 3,6 \%, ceteris paribus. 
Tabla 4.

Estimación modelo 2 sin sesgo de variable omitida por género. $M C 2 E$

\begin{tabular}{|c|c|c|c|c|}
\hline & \multicolumn{2}{|c|}{ Mujeres } & \multicolumn{2}{|c|}{ Hombres } \\
\hline Variable & Coeficiente & P-valor & Coeficiente & P-valor \\
\hline $\mathbf{C}$ & 14,07 & 0,0000 & 14.040 & 0,0000 \\
\hline EDUF & $3,33 \%$ & 0,0000 & $3,596 \%$ & 0,0000 \\
\hline EXPE & $0,50 \%$ & 0.0765 & $0,795 \%$ & 0.0031 \\
\hline EXPE^2 & $-0,01 \%$ & 0.0957 & $-0,17 \%$ & 0.0038 \\
\hline HAB & $0,28 \%$ & 0,0000 & $0,239 \%$ & 0,0000 \\
\hline RESID & $-0,63 \%$ & 0,2705 & $-0,045 \%$ & 0,4961 \\
\hline Observaciones & \multicolumn{2}{|c|}{180} & \multicolumn{2}{|c|}{390} \\
\hline R2 & \multicolumn{2}{|c|}{0,933} & \multicolumn{2}{|c|}{0,918} \\
\hline P-valor F & \multicolumn{2}{|c|}{0,0000} & \multicolumn{2}{|c|}{0,0000} \\
\hline
\end{tabular}

La experiencia prueba que dicha tasa alcanza para las mujeres $0,5 \%$ de variación por cada año, mientras que en los hombres es igual a $0,8 \%$. La variable que recoge la habilidad es mayor para las mujeres que para los hombres, lo que refleja que las profesoras tienen un mayor nivel de productividad académica que los profesores, ya que para ellas asciende a 0,28 $\%$ y para ellos a $0,24 \%$, cada vez que pasa un año. Por otro lado, cabe indicar que en ambos escenarios la variable de los residuales no es estadísticamente significativa, por lo que no existe endogeneidad.

Se aclara que los modelos anteriores por contar con información del total de la población de los docentes nombrados por la Universidad del Cauca, no tienen presencia de valores missing, por lo que no habrá sesgo de selección muestral. De manera redundante, todos los profesores están participando del mercado, hay registro de sus salarios y no se requiere la corrección de Heckman (1979).

\section{Conclusiones}

Debido a la imposibilidad de dar cumplimiento a los supuestos planteados por Mincer
(1974), no se puede calcular la tasa interna de retorno, sino más bien la tasa de crecimiento salarial frente a años adicionales de educación.

En este orden de ideas, la tasa de crecimiento salarial respecto a la educación para los docentes de la Universidad del Cauca alcanza el 3,5 \% por un año de estudio adicional. Discriminado por género se encuentra que la tasa es superior para los profesores $(3,6 \%)$ que para las profesoras $(3,3 \%)$, patrón que se mantiene para la experiencia pero no para la habilidad, mostrando que las docentes son más productivas en términos académicos que sus pares hombres.

Estos resultados no se pueden comparar directamente con los valores que se hallan en los estudios basados en la ENH (1996) para Colombia, ya que la población de profesores es más homogénea en términos de educación y actividad laboral que la población incluida en la encuesta, por lo que un año de educación en la población de profesores debe tener un efecto menor que cuando se analiza la población en su totalidad. Y en efecto, se observa que dichos valores son bastante bajos y distantes de los encontrados para todo el país e inclusive 
para el ámbito internacional. Siguiendo a Psacharopoulos y Patrinos (2004) y Prada (2006) a nivel agregado para Colombia la tasa alcanza el $14 \%$ aproximadamente y para otras naciones como Vietnam asciende a $4,8 \%$ y para Taiwán a $6,0 \%$.

Vale la pena aclarar que dichos autores encuentran que el retorno también es mayor para los hombres que para las mujeres, lo que evidencia de algún modo que las mujeres pueden estar sujetas a fenómenos de discriminación laboral, segregación, o como posiblemente sucede en el caso de las profesoras universitarias, su rol en el hogar les impide tener más chances de poder estudiar y ganar experiencia académica en comparación con los profesores.

La formación doctoral y posdoctoral en general aún es baja en los docentes de la Universidad del Cauca. Los estudios en especializaciones y maestrías son los máximos logros académicos alcanzados por ellos, lo que tal vez explique el por qué las tasas de crecimiento salarial frente a la educación no sean tan altas. La formación de los docentes de planta, la inclusión de otros educadores en el mediano y largo plazo con mayores niveles de cualificación, el relevo generacional y las exigencias mismas del entorno y del mercado, permitirán seguramente elevar dichas tasas.

La variable habilidad por productividad académica fue instrumento que ayudó a medir los retornos sin sesgo por variable omitida. Cuando no se optó por su inclusión, la tasa fue igual a 4,2\%; pero una vez se tiene en cuenta y se estima por MCO descendió a 2,61\%, resultado concordante con lo establecido por Griliches (1977), pues el resultado inicial está sobrestimado. Por último, cuando se estima por MC2E la tasa de crecimiento de los salarioseducación de nuevo asciende a 3,5\%; lo cual va en el mismo sentido de lo expuesto por Sapelli (2009), ya que se subvalora cuando se estima por MCO.
Para capturar la evolución de dicha tasa en el tiempo ya sea en el contexto nacional, regional $\mathrm{o}$ al interior de las universidades se requiere preferiblemente de información de datos panel y no de corte transversal. En efecto, la utilización de datos provenientes de la GEIH, la ECV97 o de la base de datos de las universidades, muestra la situación en un momento en el tiempo, ya que subyace la imposibilidad de hacerle un seguimiento a cada persona a través del tiempo en una región o país. En las universidades probablemente se puede lograr hacerlo por el número relativamente inferior de personas a analizar, pero esto se constituye en materia de otra investigación.

\section{Notas}

${ }^{1}$ Siguiendo a Griliches (1977), la ecuación minceriana presenta varios sesgos que complican las estimaciones por MCO. El autor identificó que el modelo evidencia variables omitidas entre las cuales se destaca la habilidad del individuo: las personas con mayor habilidad son las que deciden elegir el más alto nivel educativo, esto provocaría estimaciones inconsistentes, ya que al existir una relación directa entre el nivel educativo y la habilidad, no se cumple el supuesto de exogeneidad estricta y el resultado final es una sobrestimación de la tasa de retorno a la educación. Otro problema asociado es que la educación se encuentra medida de forma incorrecta, ya que la ecuación de Mincer implica la existencia de una única tasa de rendimiento cuando la educación debería ser desagregada y flexible para obtener un mejor resultado en la estimación de la inversión (Sapelli, 2009). Este sesgo subvalora el verdadero rendimiento. Finalmente, el tratamiento de la variable educación como exógena genera problemas, toda vez que puede estar afectada por otras variables como los costos monetarios o los costos de oportunidad de la educación. Otro inconveniente que también se liga a la ecuación de Mincer es el sesgo muestral, pues la muestra solo incluye a aquellos sujetos que están ocupados, pero prescinde de aquellos que deseaban hacerlo 
pero al momento de levantar la información no tenían empleo y por tanto no hay registro de sus salarios. Si se continúan las estimaciones sin tener en cuenta lo anterior, los parámetros bajo MCO serán sesgados e inconsistentes, ya que el modelo sufre de sesgo por variable omitida. Para corregir este nuevo obstáculo, se implementa la corrección en dos etapas de Heckman (1979).

2 La información que se suministra es anónima y contiene solo los datos de los profesores de planta (contrato indefinido) y no de los docentes catedráticos y ocasionales (contrato definido), ya que la alta volatilidad de sus contrataciones sesgaría el estudio. El salario considerado es la asignación básica mensual, y frente a la edad, se extrae en años a partir de la fecha de nacimiento del docente hasta el 30 de abril de 2014. La experiencia que se tiene en cuenta solo es la obtenida al interior de la Universidad del Cauca. Los años de educación de los profesores se estiman a partir del cúmulo de títulos alcanzados. Por último, para capturar la habilidad laboral del profesor se tuvo en cuenta su productividad académica. Es decir, los ingresos que no provienen de estudios finalizados y reconocidos, sino aquellos devengados por ascensos en el escalafón docente, publicaciones científicas en revistas indexadas, publicación de libros de texto y de ensayo, publicación de videos cinematográficos o fonográficos, patentes, traducciones, obras artísticas, premios nacionales e internacionales, ocupación de cargos administrativos (solo los que se convierten en factor salarial), innovaciones tecnológicas y software.

${ }^{3}$ Los salarios de los profesores en las universidades públicas de Colombia se rigen por el decreto 1279 de 2002 que expidió el Ministerio de Educación. Si bien es cierto el decreto contiene factores de carácter determinístico que establecen los niveles salariales, como los años de educación en posgrados, la experiencia docente e inclusive, remuneraciones temporales por bonificaciones o por la ocupación de cargos administrativos (no contemplados en el decreto en mención, pero sí en el reglamento interno de la Universidad del Cauca), existe un factor que es totalmente aleatorio (que no se recoge en la ecuación de Mincer) y corresponde a la productividad académica del docente. Los profesores más productivos (publicación de artículos en revistas indexadas, publicación de libros, patentes de inventos, entre otros), son quienes tienen los salarios más elevados y los menos productivos los más bajos. Así las cosas, los salarios dependen de factores tanto determinísticos como aleatorios y no responden a una fórmula matemática fija, lo cual implica que se requieren modelaciones econométricas para dar cuenta de su dinámica.

${ }^{4} \mathrm{La}$ experiencia que se considera es la adquirida al interior de la universidad, ya que pocos educadores son nombrados con edades avanzadas, de tal suerte que la mayoría hace su experiencia laboral y docente al interior de la misma. Además, los profesores generalmente no provienen de fuera del ámbito académico sino que se forman al interior de las universidades, iniciando como monitores o asistentes de investigación, por ejemplo.

5 El punto salarial es una unidad de medida que representa un valor monetario que determina el Ministerio de Educación año a año $\mathrm{y}$ es indexado por la inflación.

6 Como se mencionó, los salarios de los maestros en las universidades públicas de Colombia según el decreto 1279 de 2002, depende en general solo de tres factores: la experiencia docente, los años de educación y la productividad académica. Por tal razón, la productividad académica se constituye en una buena proxy de variable omitida, ya que los dos primeros factores se incluyen de manera explícita en la ecuación minceriana. Los errores aleatorios de dicha ecuación, recogen ya otros factores que por reglamento interno de la universidad, no por el decreto 1279, alzan los salarios de los profesores como por ejemplo, las remuneraciones por cargos administrativos o las bonificaciones por ponencias nacionales o internacionales. 


\section{Referencias}

Arrow, K. (1973). Higher Education As A Filter. Journal of Public Economics, 2(3), 193-216.

Becker, G. (1964). Human Capital: A Theoretical And Empirical Analysis, With Special Reference To Education. Neva York: Columbia University Press for National Bureau of Economic Research.

Becker, G. (1993). Human Capital: A Theoretical And Empirical Analysis, With Special Reference To Education. Chicago: University of Chicago Press.

Bowles, S., \& Gintis, H. (1976). Schooling in Capitalist America: Educational Reform and the Contradictions of Economic Life. Neva York: Basic Books.

Bowles, S., \& Gintis, H. (2006). Schooling in Capitalist America Revisited. Sociology of Education, 75(1), 1-18.

Cárdenas, M. (2007). Introducción a la Economía Colombiana. Bogotá: Alfaomega Colombiana.

Casas, A., Gallego, J., \& Sepúlveda, C. (2003). Retornos a la Educación y Sesgo de Habilidad: Teoría y aplicaciones en Colombia. Lecturas de Economía, 58, 69-96.

Decreto 1279 de 2002. Por el cual se establece el régimen salarial y prestacional de los docentes de las Universidades Estatales. Diario Oficial No. 44.840. Presidencia de la República, junio de 2002.

Doeringer, P., \& Piore, M. (1971). Internal Labour Markets and Manpower Analysis. Nueva York - Londres: M. E. Sharpe Inc.

Doeringer, P., \& Piore, M. (1983). Labor Market Segmentation; To what Paradigm
Does It Belong?. American Economic Review, 73(2), 249-253.

García, F., Guataqui, J., Guerra, J., \& Maldonado, D. (2009). Beyond The Mincer Equation: The Internal Rate of Return to Higher Education in Colombia (Universidad Del Rosario, Documentos de Trabajo No. 005745). Recuperado de http://www. urosario.edu.co/economia/documentos/ pdf/dt68.pdf

Griliches, Z. (1977). Estimating the Returns to Schooling: Some Econometric Problems. Econometrica, 45, 1-22.

Hausman, J. (1978). Specification Test in Econometrics. Econometrica, 46(6), 1251-1271.

Heckman, J. (1979). Sample Selection Bias as a Specification Error. Econometrica, 47(1), 153-161.

Heckman, J., Lochner, L., \& Todd, P. (2005). Earnings Functions, Rates Of Return, And Treatment Effects: The Mincer Equation And Beyond (National Bureau of Economic Research -[NBER], Working Paper Series. No. 11544). Recuperado de http://www.nber.org/papers/w11544.pdf

Johansen, S. (1992). Determination of Cointegration Rank in the Presence of a Linear Trend. Oxford Bulletin of Economics and Statistics, 54(3), 383-397.

Maldonado, D. (2000). Diferencias Salariales Entre los Trabajadores del Sector Público y del Sector Privado en Colombia en 1997. Desarrollo y Sociedad, 45, 65-106.

Mincer, J. (1974). Schooling, Experience, And Earnings. New York: Columbia University Press.

Organización para la Cooperación y el Desarrollo-[OCDE]. (1998). Human Capital 
Investment: An International Comparision. Francia: Center For Educational Research and Innovation.

Prada, C. (2006). ¿Es rentable la Decisión de Estudiar en Colombia?. Ensayos Sobre Política Económica, 51, 226-323.

Psacharopoulos, G., \& Patrinos, H. (2004). Returns to Investment in Education: A Further Update. Education Economics, 12(2), 111-134.

Psacharopoulos, G., \& Vélez, E. (1992). Educación, Habilidad e Ingresos en Colombia, 1988. Planeación y Desarrollo, 23(2), 74-104.

Reich, M., Gordon. M., \& Edwards, R. (1973). Dual labor Markets: A Theory of Labour Market Segmentation. American Economic Review, 63, 359-65.

Salas, M. (2008). Economía de la Educación. Madrid: Pearson.

Sapelli, C. (2009). Los Retornos a la Educación en Chile: Estimaciones por Corte Transversal y por Cohortes (Pontificia Universidad Católica de Chile Documento de Trabajo $N^{\circ} 349$ ).
Schultz, T. (1959). Investment in Man: An Economist's View. Social Service Review, 33, 109-117.

Spence, M. (1973). Job Market Signaling. Quarterly Journal of Economics, 87(3), 355-374.

Stiglitz, J. (1975). The Theory of "Screening", Education, and Distribution of Income. American Economic Review, 65(3), 283300.

Thurow, L. (1975). Generating Inequality: Mechanisms of Distribution in the U.S. Economy. Nueva York: Basic Books.

Thurow, L. (1983). Education and Economic Equality. The Public Interest, 28, 66-81.

Tenjo, J. (1993). Educación, Habilidad, Conocimientos e Ingresos. Planeación y Desarrollo, 24, 85-102.

Vargas, B. (2013). Retornos a la Educación y Migración Rural-Urbana en Colombia. Desarrollo y Sociedad, 72, 205-223.

Wooldridge, J. (2001). Introducción a la Econometría: Un enfoque Moderno. México: Thompson Learning. 
